\title{
Mastering the Dilemma of Rare Diseases
}

The field of pediatric neurology comprises an abundance of rare diseases, many of which are bizarre and most are incurable. This situation is a dilemma for doctors and patients alike. For a physician it is impossible to memorize and recognize all these strange conditions. For patients and families it is a disaster when, after great effort, a diagnosis is eventually reached that nobody, neither friends nor the family doctor, have ever heard of before. The rarity of the condition alone causes a particular type of grief - how can such a terrible oddity happen to us! Scientific progress is also hampered by the rarity of a disease with the consequence that no cures are being developed, and not even existing knowledge of palliative therapies and counselling can be properly applied in an individual case.

In this issue of Neuropediatrics, a short article [1] on a rare genetic disorder highlights the route the medical and scientific community should take when confronted with the dilemma of very uncommon diseases. The paper presents the clinical and genetic findings in two Italian brothers suffering from CLN5, a variant form of late infantile neuronal ceroid lipofuscinosis (NCL) that, until recently, was thought not to occur outside Finland. The merits of the study are twofold. As a medical report, the paper is a nice example of how to study a rare case in a multidisciplinary way and to present the results concisely with a high data/text ratio. As a contribution for practicing pediatricians at large, the study enlarges our diagnostic horizon when confronted with a young child suffering from a severe, unexplained epilepsy and developmental standstill. In the realm of neurodegenerative diseases of the CNS that become manifest during childhood or adolescence, the NCL are the most common group. They are characterized by a combination of epilepsy, dementia and visual loss through retinal degeneration. To date, 10 different genetic forms of NCL have been defined and can be diagnosed, among them forms that begin in the neonatal, infantile, late infantile, juvenile, or adult periods of life. For some of them, inexpensive diagnostic screening tests are available, for other forms the procedures include costly molecular genetic and electron microscopic studies, and for a small group of poorly known NCL forms a real scientific project is necessary $[2,3]$.

At this point, our willingness to invest a reasonable effort in the diagnosis and management of such a rare and difficult case is in danger. What is the way out of the dilemma? When we cannot rely on our own expertise, firing some diagnostic buckshot may be inefficient and expensive. So we seek advice from other experts, and in such situations many of us nowadays turn to the internet. There, however, we may be overwhelmed by a huge flood of information, the suitability of which for the individual case is frequently questionable. We may find guidelines issued by professional or lay organizations, but what we would really like to have is a specifically qualified expert network where a real person will answer questions from doctors and patients by e-mail or phone or will refer us to other specialists. Such services do exist for a number of rare disorders, but are not easy to find. With time, a whole system of such networks, including procedures for easy orientation and accessibility, will have to be developed. For the NCL disorders, one of the existing services (www.ncl-net.com) is currently being extended for cooperative worldwide use. It appears to be the responsibility of professional societies and journals to foster such expert networks for rare diseases, to develop criteria for judging their quality and usefulness, and to keep us informed about them. Then also the gain of knowledge from a short case report has an optimal chance to reach those who need it.

Prof. A. Kohlschuetter, MD

\section{References}

1 Cannelli N, Nardocci N, Cassandrini D, Morbin M, Aiello C, Bugiani M, Criscuolo L, Zara F, Striano P, Granata T, Bertini E, Simonati A, Santorelli F. Revelation of a novel CLN5 mutation in early juvenile neuronal ceroid lipofuscinosis. Neuropediatrics 2007; 38: 45-48

2 Mole SE, Williams RE, Goebel HH. Correlations between genotype, ultrastructural morphology and clinical phenotype in the neuronal ceroid lipofuscinoses. Neurogenetics 2005; 6: 107-126

3 Siintola E, Lehesjoki A-E, Mole SE. Molecular genetics of the NCLs - status and perspectives. BBA Mol Basis Dis 2006; 1762: 857-864

\section{Prof. A. Kohlschuetter, MD}

Department of Pediatrics Neurochemistry \& Metabolism University Medical Center Eppendorf

Martinistr. 52

20246 Hamburg

Germany

Tel.: + 49/40/428 034300/6391

Fax: $+49 / 40 / 428035137$

kohlschuetter@uke.uni-

hamburg.de 